\title{
Role of diet on intestinal metabolites and appetite control factors in SD rats
}

\author{
BO LIN ${ }^{1}$, YUEMING LIU $^{1}$, WEI ZHANG ${ }^{1}$ and WENLI ZOU ${ }^{1,2}$ \\ ${ }^{1}$ Department of Nephrology, Zhejiang Provincial People's Hospital; ${ }^{2}$ Department of Nephrology, \\ People's Hospital of Hangzhou Medical College, Hangzhou, Zhejiang 310014, P.R. China
}

Received August 14, 2018; Accepted May 31, 2019

DOI: $10.3892 /$ etm.2020.8993

\begin{abstract}
The present study aimed to investigate changes in the levels of metabolites and appetite control factors caused by different dietary interventions in Sprague Dawley (SD) rats. A total of 35 male SD rats were weaned and immediately randomly assigned to five groups. The control group was given ad libitum access to a normal chow diet, and the other groups received a high-fat diet (FAT group), high-sugar diet, high-fibre or high-protein diet (PRO group) for 4 weeks. The high-fat diet contributed to weight gain and adipose tissue formation, and affected lipid indexed. The FAT group had a higher body weight, Lee's index, adipose mass and glucose tolerance than all of the other groups. The opposite effect was observed in the PRO group. High-performance liquid chromatography revealed that short-chain fatty acid and amino acid formation were affected by the various diets. In addition, differences in the mRNA expression levels of leptin, ghrelin and associated receptors were determined in the gastrointestinal, adipose and hypothalamus tissues. The present study provides further evidence of the role of diet in obesity development and prevention. It also highlights the role of intestinal metabolites and appetite control factor expression in the pathogenesis of obesity in SD rats.
\end{abstract}

\section{Introduction}

The prevalence of obesity has increased; according to the World Health Organization, $>1.9$ billion adults were overweight in 2014 , and of these, $>600$ million were obese (1). Increased energy intake or decreased physical activity are the two most obvious contributing factors. The type of diet has an important role in excessive caloric intake and excessive body fat accumulation. Recently, the gut microbiota has been implicated in the

Correspondence to: Dr Wenli Zou, Department of Nephrology, Zhejiang Provincial People's Hospital, 158 Shangtang Road, Hangzhou, Zhejiang 310014, P.R. China

E-mail: 554138648@qq.com

Key words: diet, obesity, intestinal microbiota, short-chain fatty acid, amino acid, leptin etiology of obesity (1). The diet and intestinal microbiota have crucial effects on health, since they are involved in nutritional supply, immunology and tumorigenesis (2).

Intestinal metabolites are produced by diet catabolism and bacterial metabolism $(3,4)$. Food is processed through physical and chemical breakdown in the digestive tract, producing nutrients to be absorbed. Most nutrients cannot be absorbed without the presence of the intestinal microbiota. The intestinal microbiota provides an indispensable array of enzymes that degrade complex dietary substrates. Different metabolites have varying roles in disease development. According to current studies, microbial metabolism of dietary carbohydrates results mainly in the formation of short-chain fatty acids (SCFAs) and gases, while the production of acetate, propionate and butyrate take part in regulating the intestinal barrier, bone mass and central nervous system autoimmunity (5-7).

The consumption of different diets not only provides substrates for the body's metabolism, but also affects the structure and metabolism of the microbial community $(2,3)$. The post-weaning role of the dietary composition in shaping the adult microbiome composition is of particular importance. The amount, type and balance of the major dietary macronutrients (carbohydrates, proteins and fats) have a considerable effect on the large intestinal microbiota $(3,8)$. Diet is a major factor that shapes the composition and activity of enteric dysbacteriosis $(9,10)$. Effective manipulation of the gut microbiota by altering dietary patterns has been proved to have the potential to prevent metabolic disorders by numerous studies (8). Unfortunately, little is known about how diet and intestinal bacteria affect cellular health.

Owing to the complexity of the microbiota, interactions of the intestinal bacteria with the host are difficult to study. In recent years, high-throughput sequencing technology has been used to study the intestinal flora, and the components of the fecal flora have been almost entirely elucidated $(11,12)$. However, this technology has limitations in testing for bacterial genera and species, and the same species of bacteria have numerous different effects at the subspecies levels. Since intestinal bacteria cannot penetrate the gastrointestinal tract, the present study hypothesizes that the possible molecular mechanisms associated with obesity rely on intestinal metabolites.

In the present study, the levels of intestinal metabolites and expression of appetite control factors were assessed in Sprague Dawley (SD) rats fed different diets. The present study aimed 
to explore the role of intestinal metabolites in obesity development and prevention from a novel perspective.

\section{Materials and methods}

Animals and diets. The present study was approved and monitored by the Ethics Committee of the Second Xiangya Hospital of Central South University (Changsha, China), where the animal experiment was performed, and the protocol was in accordance with the guiding principles covered in the Guide for the Use and Care of Experimental Animals. All surgical procedures were performed under chloral hydrate anesthesia and all efforts were made to minimize the suffering of the experimental animals. SD rats of 5-week-old (Hunan SJA Laboratory Animal Co., Ltd.) weighing 62.1-102.3 g with the same genetic background were used for all the experiments. After a 1-week acclimation, all 35 male specific pathogen-free SD rats were weaned and immediately randomly assigned to the FAT, SUG, FIB, PRO and control diet (CON) groups (seven rats in each). The CON group was provided ad libitum access to a normal chow diet, while the FAT, SUG, FIB and PRO groups were fed a high-fat, high-sugar, high-fibre and high-protein diet, respectively, for 4 weeks. Protein, fat, sugar and fibre are responsible for $60 \%$ of the calories in the PRO, FAT, SUG and FIB diet group, respectively. All of the diets are listed in Table I. Water and food were provided ad libitum and the body weights were recorded weekly. At the end of the feeding protocol, body composition analysis and oral glucose tolerance test (OGTT) were performed as described below. After 4 weeks, all the rats were sacrificed, blood samples were collected and the contents of the cecum and stool samples, as well as fat, gastric, intestinal, liver and brain tissues were harvested.

Measurements of body length and weight, and Lee's index. The animals were weighed prior to feeding weekly, and the weight change trend of the five groups was monitored. Furthermore, the body length and the final weight of the rats were measured under anesthesia, prior to euthanasia. Lee's index was calculated using the following formula: Lee's index $=. \quad \sqrt[3]{\text { weight }(\mathrm{g}) \mathrm{x} 1,000 \div \text { length }(\mathrm{cm})}$

Measurements ofbody fat andfatindex. The fat tissue (omental-, perirenal- and peritesticular adipose fat) was harvested and measured, and the fat index was then calculated as follows: Fat index $(\%)=[$ fat content $(\mathrm{g}) /$ body weight $(\mathrm{g})] \mathrm{x} 100$.

Fasting blood glucose (FBG) and OGTT. After a 12-h fast, blood was collected from the tail vein and FBG was measured using a glucose meter (LifeScan). Subsequently, glucose ( $2 \mathrm{~g} / \mathrm{kg}$ rat) was administered intragastrically, and blood glucose was determined again at 30, 60, 90 and $120 \mathrm{~min}$ post-administration via tail.

Biochemical analysis. At the end of the experiment, all of the animals were anesthetized by intraperitoneal injection of $10 \%$ chloral hydrate $(400 \mathrm{mg} / \mathrm{kg})$ after a 12 -h overnight fast. Blood samples $(\sim 1.0 \mathrm{ml})$ were collected via cardiac puncture and centrifuged $\left(4^{\circ} \mathrm{C} ; 3,000 \mathrm{r} / \mathrm{min} ; 15 \mathrm{~min}\right)$ to obtain the serum. The concentrations of serum triglyceride (TG), high-density lipoprotein cholesterol (HDL-C) and low-density lipoprotein cholesterol (LDL-C) were analyzed using an automatic biochemical analyzer provided by The Second Xiangya Hospital of Central South University (Changsha, China). The levels of serum leptin and ghrelin were assayed using the rat leptin ELISA kit (cat. no. ELR-Leptin-001; RayBiotech) and the rat ghrelin enzyme immunoassay kit (cat. no. EIA-GHR-1; RayBiotech), respectively.

Reverse transcription-quantitative $(R T-q) P C R$. Samples of fat, gastric, intestinal, liver and brain tissues were collected and stored in dry ice $\left(-80^{\circ} \mathrm{C}\right)$ for further evaluation. The RNA of the tissues were extracted using TRI reagent (Takara Bio. Inc.). RT was performed using a PrimeScriptÔ RT reagent kit with gDNAEraser (Takara Bio. Inc.) using the appropriate primers. Levels of the genes of interest were determined using qPCR with SYBR Green master mix (cat. no. RR820A; Takara Bio. Inc) using Step One Plus equipment (Bio-Rad CFX96T $\mathrm{M}$ real-time system, C1000 touch thermal cycle; Bio-Rad Laboratories). The thermocycling conditions were as follows: $95^{\circ} \mathrm{C}$ for $30 \mathrm{sec}$; PCR (50 cycles), denaturation $5 \mathrm{sec}$ at $95^{\circ} \mathrm{C}$, annealing for $30 \mathrm{sec}$ at $60^{\circ} \mathrm{C}$; denaturation at $95^{\circ} \mathrm{C}$ for $15 \mathrm{sec}$, annealing at $6^{\circ} \mathrm{C}$ for $1 \mathrm{~h}$, heating at $95^{\circ} \mathrm{C}$ for $10 \mathrm{sec}$. The gene expression was normalized to that of endogenous expression of the genes of interest and respective primers are listed in Table II. The $2^{-\Delta \Delta \mathrm{Cq}}$ method was used for quantification of data $(13,14)$.

Pre-column derivatization reverse-phase high-performance liquid chromatography (RP-HPLC). The HPLC system used was an LC-20A HPLC system (Shimadzu Corp.) equipped with a Shimadzu LC-20AD dual pump, Rf-10ADvp fluorescence detector (Shimadzu Corp.), manual injection port (20 $\mu \mathrm{l})$ and LabSolution/LCSolution Lite chromatography Chemstation (Shimadzu Corp.). The separation was performed using a Thermo Hypersil C18 column (length, $150 \mathrm{~mm}$; internal diameter, $4.6 \mathrm{~mm}$; particle size, $4 \mu \mathrm{m}$; Shimadzu Corporation) by using a linear gradient elution mode at a flow rate of $1 \mathrm{ml} / \mathrm{min}$. All reagents used in the present study were of analytical grade unless otherwise specified.

Determination of SCFAs. In the present study, a pre-column derivatization method was used to determine the SCFAs using RP-HPLC. The samples were first acid-extracted by adding $1 \mathrm{mg}$ of the feces or ileocecal content samples to $1 \mathrm{ml}$ $12 \%$ sulfuric acid or $0.5 \mathrm{ml}$ of blood sample to $0.5 \mathrm{ml} 12 \%$ sulfuric acid and vortexing ( $2 \mathrm{~min}$ ), followed by ultrasonic processing (35 kHz; $10 \mathrm{~min}$ ). Subsequently, the mixture was added to $2 \mathrm{ml}$ methyl tertiary butyl ether (Sinopharm Chemical Reagent Co., Ltd), and after extraction and centrifugation $\left(8,000 \mathrm{x} \mathrm{g} ; 4^{\circ} \mathrm{C} ; 5 \mathrm{~min}\right)$, the upper organic phase was collected, mixed with $20 \mu 150 \mathrm{mmol} / \mathrm{l}$ potassium hydroxide-methanol solution, and then dried under a nitrogen stream. Standard solutions of the SCFAs (chromatographic grade; Supelco) formic, acetic, propionic, butyric, isobutyric, malic, valeric, lactic and succinic acid were accurately diluted to obtain a series of mixed reference solutions of various concentrations using acetonitrile (chromatographic grade; TEDIA Co.). The derivative reagent was 2,4'-dibromoacetophenone (chromatographic grade; Sigma-Aldrich; Merck KGaA) and 18-crown-6 
Table I. Composition $(\mathrm{g} / \mathrm{kg})$ and energy density of the five diets.

\begin{tabular}{|c|c|c|c|c|c|}
\hline Component & Normal control & High-protein & High-fibre & High-sugar & High-fat \\
\hline Casein & 200 & 619.9 & 181.8 & 200 & 271.9 \\
\hline Corn starch & 547.0 & 122.6 & 377.2 & 75.5 & 133.2 \\
\hline Dextrin & 0 & 0 & 120.0 & 0 & 0 \\
\hline Sucrose & 100 & 99.3 & 90.9 & 571 & 135.9 \\
\hline Soy oil & 70 & 69.5 & 63.6 & 70 & 345.2 \\
\hline Fibre & 50 & 49.6 & 90.9 & 50 & 68.0 \\
\hline Pectin & 0 & 0 & 45.5 & 0 & 0 \\
\hline Minerals & 27.3 & 27.1 & 24.8 & 27.3 & 37.1 \\
\hline Vitamins & 0.26 & 0.26 & 0.2 & 0.26 & 0.35 \\
\hline L-cystine & 3 & 9.3 & 2.7 & 3 & 4.1 \\
\hline Choline chloride & 2.5 & 2.5 & 2.3 & 2.5 & 4.2 \\
\hline TBHQ & 0.014 & 0.014 & 0.013 & 0.014 & 0.07 \\
\hline Energy density (kcal/kg) & 3,810 & 3,810 & 3,528 & 3,810 & 5,179 \\
\hline
\end{tabular}

TBHQ, tert-butylhydroquinone.

Table II. Sequences of primers used for PCR.

\begin{tabular}{llclc}
\hline Primer & \multicolumn{1}{c}{ Forward (5'-3') } & Bp length & \multicolumn{1}{c}{ Reverse (3'-5') } & Bp length \\
\hline Leptin & ACCTGGAGAACCTGCGAGAC & 20 & TAGAGGAGTAGGAGAAACGGACA & 23 \\
Leptin receptor & ACCCAGCACAATCCAATCACTA & 22 & TTGAGCTCTGATGTAGGACGAATAG & 25 \\
Ghrelin & GCCACTCTGGGTGTTCTTTTG & 21 & GCAGATGA GATGGGTCTTTATTG & 23 \\
Ghrelin receptor & GCTGGTCATCCTTGTCATCTG & 21 & TTCACTGTCTGCTTGTGGTTCT & 22 \\
$\beta$-actin & GGAGATTACTGCCCTGGCTCCTA & 23 & GACTCATCGTACTCCTGCTTGCTG & 24 \\
\hline
\end{tabular}

(chromatographic grade; Sigma-Aldrich; Merck KGaA) was used as a catalyst for the derivatization reaction. A Thermo Hypersil C18 column (length, $150 \mathrm{~mm}$; internal diameter, $4.6 \mathrm{~mm}$; particle size, $4 \mu \mathrm{m}$ ) was used at $35^{\circ} \mathrm{C}$, and the mobile phase consisted of a methanol-water $(75: 25$, v/v) with a flow rate of $1.0 \mathrm{ml} / \mathrm{min}$. The detection wavelength was $255 \mathrm{~nm}$.

Determination of amino acids. In the present study, a pre-column derivatization method was used to determine the amino acids using RP-HPLC. The feces or ileocecal samples were first acid-extracted by adding $1 \mathrm{mg}$ to $1 \mathrm{ml} 0.1 \mathrm{~mol} / 1$ hydrochloric acid or $100 \mu 1$ of the blood samples to $100 \mu 1$ anhydrous ethanol, followed by centrifugation for $5 \min \left(4^{\circ} \mathrm{C}\right.$; $8,000 \mathrm{x} \mathrm{g}$ ). Standard solutions of the following amino acids (thin layer chromatography grade; Sigma-Aldrich; Merck $\mathrm{KGaA}$ ), aspartic acid, glutamic acid, histidine, glycine, serine, threonine, arginine, alanine, tyrosine, methionine, valine, phenylalanine, isoleucine, leucine and lysine were confected into a $1.00 \mathrm{mmol} / 1$ solution by adding $0.1 \mathrm{~mol} / 1$ hydrochloric acid accurately, and the stock was then diluted to obtain a series of mixed reference substance solutions of varying concentrations using double-distilled water. The derivative reagent was a mixture of $10 \mathrm{mg}$ o-phthalaldehyde (chromatographic grade; Sigma-Aldrich; Merck KGaA), 0.6 ml methyl alcohol (chromatographic grade, Bcl International Trading
Co., Ltd.), $2.4 \mathrm{ml} 2 \%$ boric acid and $30 \mu \mathrm{l}$ mercaptoethanol (Sigma-Aldrich; Merck KGaA). A Thermo Hypersil C18 column $(150 \times 4.6 \mathrm{~mm}, 4 \mu \mathrm{m})$ was used at $35^{\circ} \mathrm{C}$ with a mobile phase consisting of solution A (a mixture of $160 \mathrm{ml}$ methyl alcohol, $900 \mathrm{ml}$ sodium acetate and $10 \mathrm{ml}$ tetrahydrofuran) and solution B (a mixture of $800 \mathrm{ml}$ methyl alcohol, $200 \mathrm{ml}$ sodium acetate and $10 \mathrm{ml}$ tetrahydrofuran) run at a flow rate of $1.0 \mathrm{ml} / \mathrm{min}$. The emission and excitation wavelengths for detection were 430 and $338 \mathrm{~nm}$, respectively.

Statistical analysis. All values are expressed as the mean \pm standard error and the statistical analyses were performed using SPSS version 22.0 (IBM Corp.). Values were compared among the five groups using analysis of variance (ANOVA), and a post-hoc least-significant differences test was performed when ANOVA indicated significance. $\mathrm{P}<0.05$ was considered to indicate a statistically significant difference.

\section{Results}

Test diets have a significant role in initiating metabolic disorders. In the present study, the SD rats were provided with different diets ad libitum for 4 weeks. The abdominal anatomy of representative animals from the five groups is presented in Fig. 1A-E. The FAT group had more abdominal 


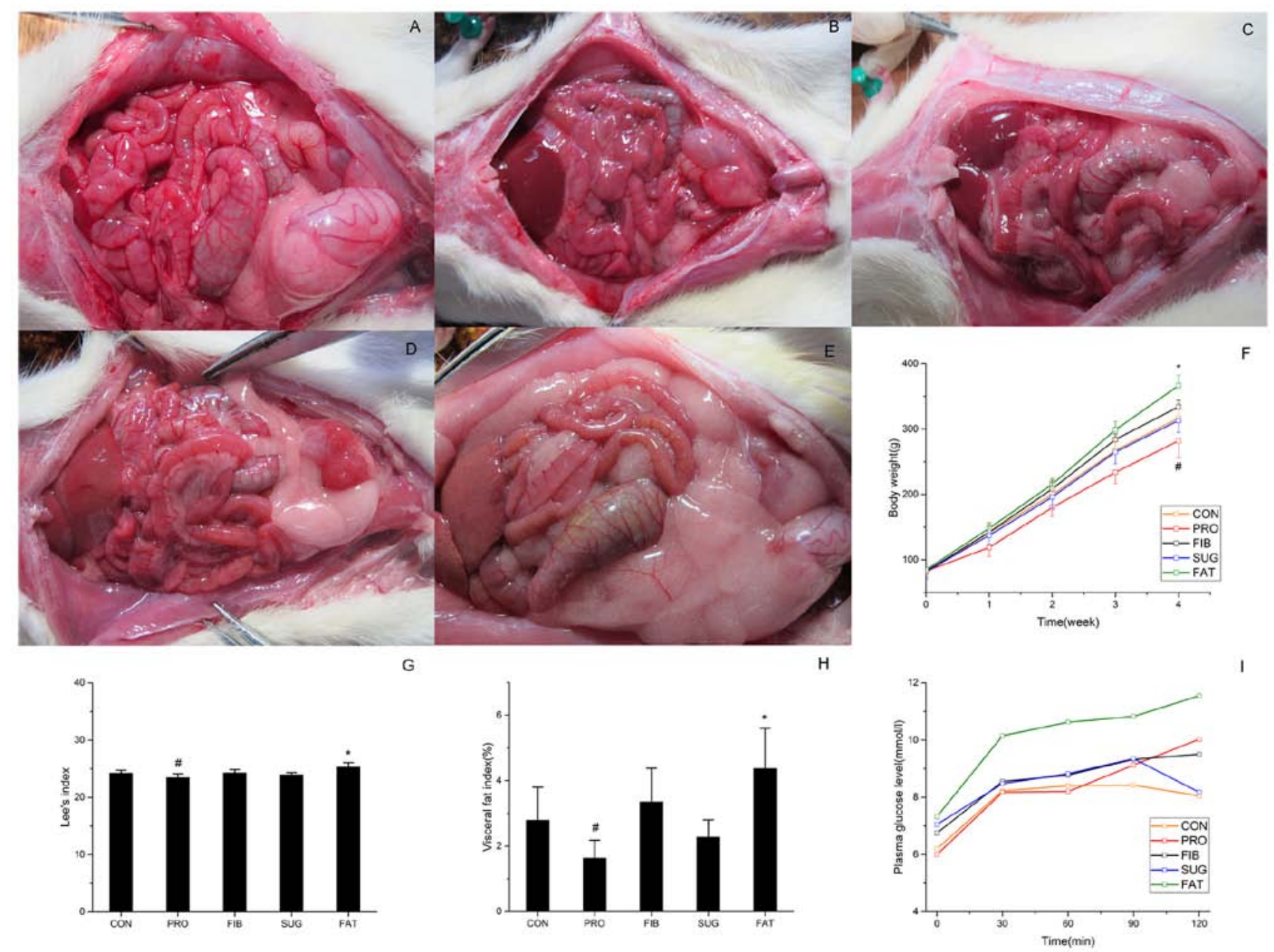

Figure 1. Abdominal anatomy and nutritional indicators in rats. (A-E) Features of abdominal anatomy of the five groups are listed as follows: (A) CON, (B) PRO, (C) FIB, (D) SUG and (E) FAT group. (F) Body weight, (G) Lee's index, (H) visceral fat index and (I) oral glucose tolerance. "P $<0.05$ FAT group vs. CON group. ${ }^{~} \mathrm{P}<0.05$ PRO group vs. CON group. Groups: CON, normal control diet; PRO, high-protein diet; FIB, high-fibre diet; SUG, high-sugar diet; FAT, high-fat diet.

fat accumulation and fatty livers (the liver color turned yellow and had a dull appearance). The animals from the PRO group had a lean body. The body weight, Lee's index, fatty content, lipids and glucose index were also analyzed. As presented in Fig. 1F, the body weight of each group exhibited a linear increase over time. At the beginning of the experiment, no significant difference in body weight was present between the groups. At the end of the experiment, the FAT group exhibited a significantly greater weight increase and fat accumulation, and developed obesity when compared to the CON group. However, the PRO group had a significantly lower weight, whereas the high-fibre and high-glucose diets did not lead to any significant changes compared with the control diet. There was a positive association among the trends regarding Lee's index, visceral fat index and body weight. As presented in Fig. $1 \mathrm{G}$ and $\mathrm{H}$, respectively, Lee's index and the visceral fat index of the PRO group was significantly lower than that of the CON group. However, the values in the FAT group were significantly higher than those in the CON group. Fig. 1I presents the changes in glucose levels over time. The high-fat diet elevated the FBG levels, and delayed the glucose level decrease in the OGTT, the glucose level at $2 \mathrm{~h}$ post-glucose exposure was $>11 \mathrm{mmol} / 1$. Compared with that in the CON group, the glucose level was also elevated in the FIB and PRO groups. Furthermore, various lipid indexes (TG, HDL-C and LDL-C) were compared among the five groups, indicating that the TG levels were significantly decreased in the PRO group. However, the FAT group exhibited a significant elevation compared with the other groups. Furthermore, the HDL-C level was significantly decreased in the FAT group and the LDL-C level was significantly elevated in the SUG group (Table III). Therefore, the high-fat diet led to impaired glucose tolerance and accumulated fat, while the high-protein diet exhibited weight loss compared with the CON group.

Potential mechanisms of obesity assessed using metabolomics. SCFAs and amino acids are important nutrients taking part in energy metabolism and regulation. In the present study, the contents of SCFAs and amino acid in the feces, ileocecal content, and blood samples were analyzed using HPLC analysis and compared.

SCFAs. As presented in Fig. 2A, the total content of SCFAs in the feces, ileocecal content and blood samples differed among the five groups. In the fecal samples, the contents of total SCFAs were successively reduced in the FAT, FIB, SUG, CON 
A

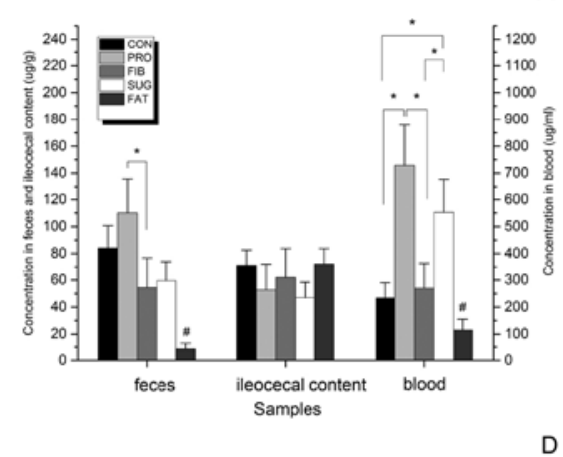

D

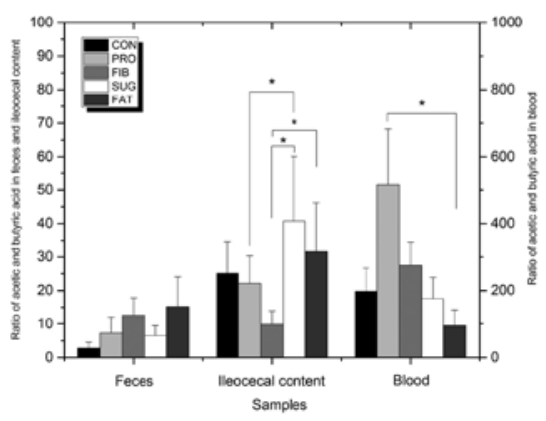

G

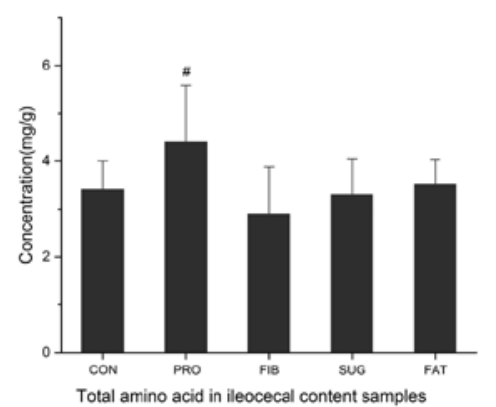

J
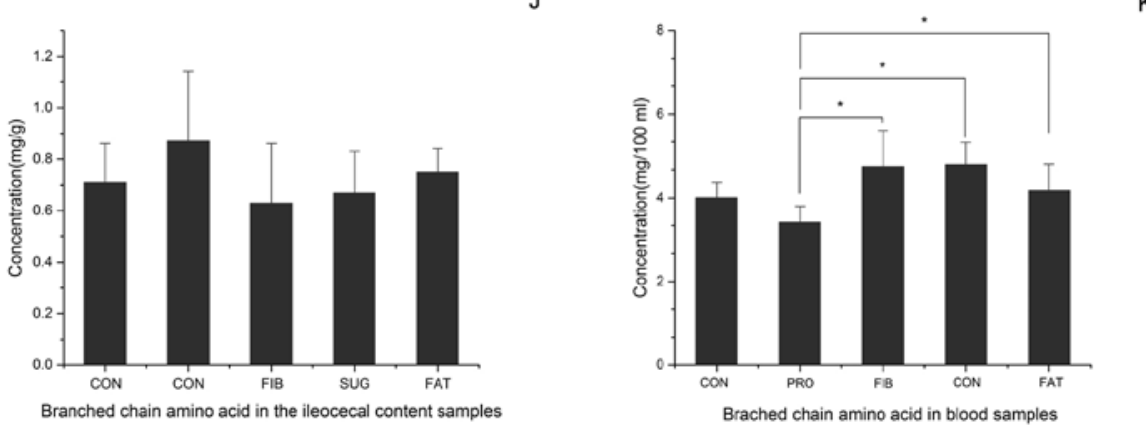

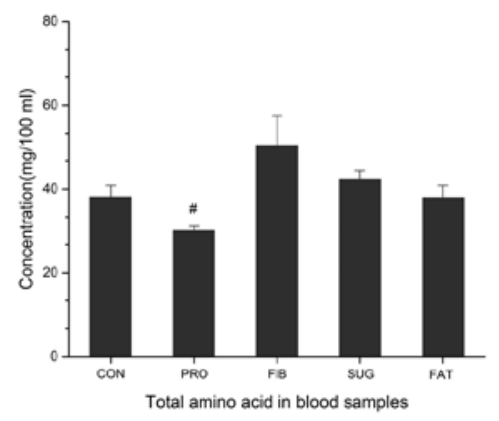

B
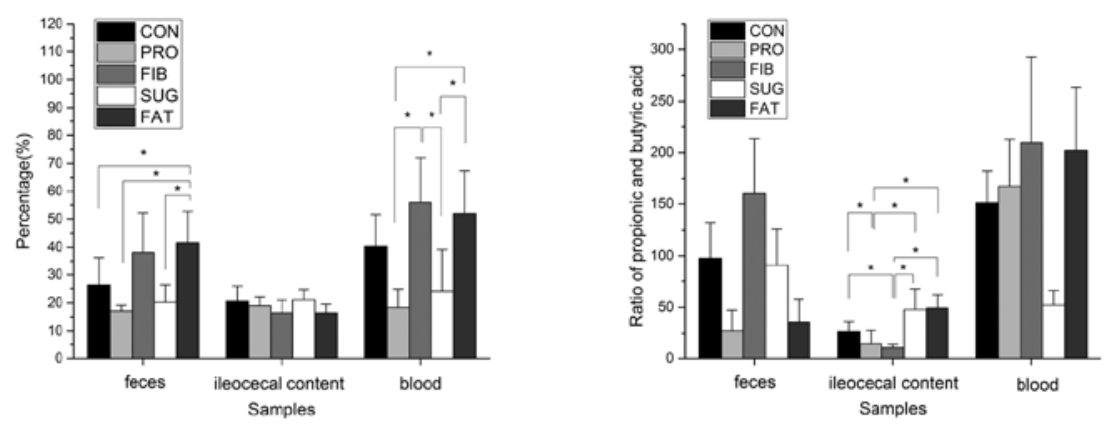

E
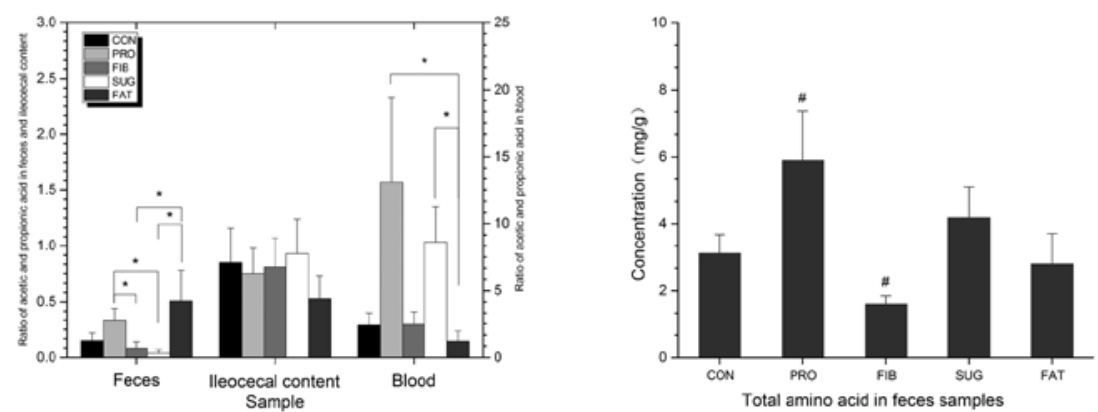

H

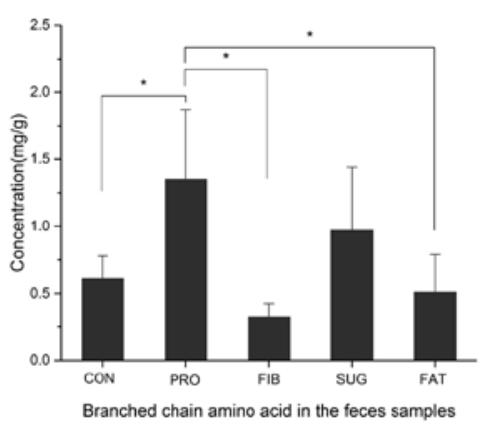

Figure 2. Comparison of the content of SCFAs and AAs among different samples of the five groups. (A) Total SCFA concentration, (B) content of acetic, propionic and butyric acid, (C) acetic acid/propionic acid ratio, (D) acetic acid/butyric acid ratio and (E) propionic acid/butyric acid ratio in different samples. (F-H) Total content of AAs in (F) feces, (G) ileocecal content and (H) blood samples, respectively; (I-K) Content of branched chain amino acids in (I) feces, (J) ileocecal content and (K) blood samples, respectively. ${ }^{*} \mathrm{P}<0.05$ vs. two assigned groups, ${ }^{~} \mathrm{P}<0.05$ vs. one assigned group compared with the other groups. Groups: CON, normal control diet; PRO, high-protein diet; FIB, high-fibre diet; SUG, high-sugar diet; FAT, high-fat diet. SCFA, short-chain fatty acids; AA, amino acids.

and PRO group, and the FAT group exhibited a significant decrease compared with the other groups. In addition, there was a significant difference between the FIB and PRO groups. No difference between the groups was identified in the ileocecal content. In the blood samples, the content in the FAT group samples was significantly decreased compared with that in the all other groups, the PRO and the SUG group exhibited a significant increase when compared with the CON, FIB and FAT groups, while there was no significant differences exhibited between the PRO and SUG group. Acetic acid, propionic acid and butyric acid are popular SCFAs, and to eliminate the influence of sample wetness, the percentage of the three SCFAs among the total SCFAs in each sample was analyzed (Fig. 2B). The feces samples of the FAT group had significantly elevated 


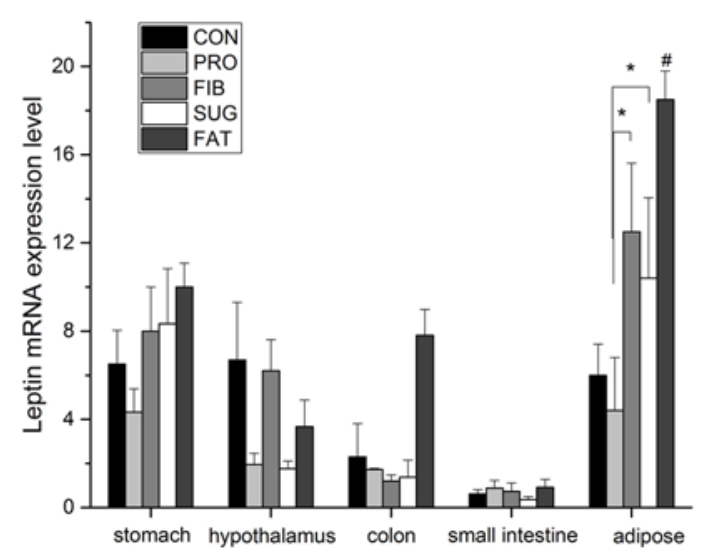

Leptin levels in different tissues of different experimental groups

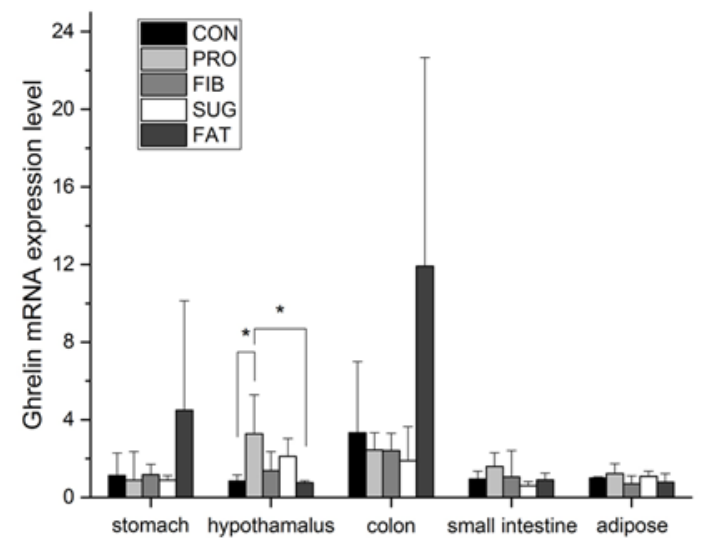

Ghrelin levels in different tissues of different experimental groups
A

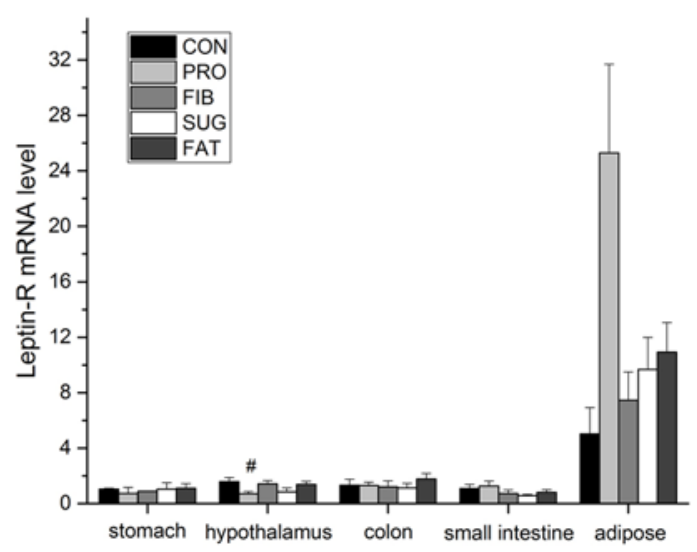

Leptin-R levels in different tissues of different experimental groups

C

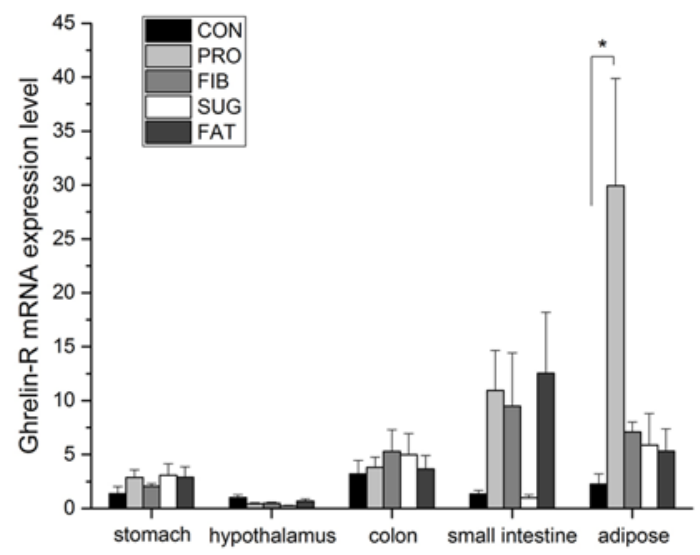

Ghrelin-R levels in different tissues of different experimental groups

Figure 3. Leptin and ghrelin expression in different tissues of the five groups. (A) Leptin mRNA levels; (B) leptin receptor mRNA levels; (C) ghrelin mRNA levels; (D) ghrelin receptor mRNA levels. " $\mathrm{P}<0.05$ vs. two assigned groups, ${ }^{\prime} \mathrm{P}<0.05$ vs. one assigned group compared with the other groups. Groups: CON, normal control diet; PRO, high-protein diet; FIB, high-fibre diet; SUG, high-sugar diet; FAT, high-fat diet.

Table III. Comparison of serum lipid levels in the different experimental groups.

\begin{tabular}{llcc}
\hline Group & TG (mmol/l) & HDL-C $(\mathrm{mmol} / \mathrm{l})$ & LDL-C $(\mathrm{mmol} / \mathrm{l})$ \\
\hline CON & $0.818 \pm 0.028$ & $1.268 \pm 0.017$ & $0.185 \pm 0.024$ \\
PRO & $0.353 \pm 0.120^{\mathrm{a}}$ & $1.230 \pm 0.476$ & $0.228 \pm 0.048$ \\
FIB & $0.738 \pm 0.152$ & $1.295 \pm 0.031$ & $0.190 \pm 0.334$ \\
SUG & $0.930 \pm 0.036$ & $1.220 \pm 0.036$ & $0.253 \pm 0.032^{\mathrm{b}}$ \\
FAT & $1.420 \pm 0.364^{\mathrm{c}}$ & $1.098 \pm 0.045^{\mathrm{d}}$ & $0.220 \pm 0.042$ \\
\hline
\end{tabular}

${ }^{\mathrm{a}} \mathrm{P}<0.01,{ }^{\mathrm{b}} \mathrm{P}<0.05,{ }^{\mathrm{c}} \mathrm{P}<0.001,{ }^{\mathrm{d}} \mathrm{P}<0.001$ vs. $\mathrm{CON}$ group. Values are expressed as the mean \pm standard deviation $(7$ animals per group). Groups: CON, normal control diet; PRO, high-protein diet; FIB, high-fibre diet; SUG, high-sugar diet; FAT, high-fat diet. TG, triglycerides; H/LDL-C, high/low-density lipoprotein cholesterol.

levels than the other groups except the FIB group. The blood samples of the FAT and FIB group had significantly elevated levels than the PRO group and SUG group. The propionic acid/butyric acid ratio of the feces and blood samples did not differ among the five groups (Fig. 2C). The ileocecal content of the PRO and FIB groups exhibited significantly decreased ratios of propionic acid/butyric than the other groups, but there was no differences exhibited between the PRO and FIB group. Comparing the acetic acid/butyric acid ratio in the feces samples of the various groups, no significant differences were identified. In the ileocecal content, the acetic acid/butyric acid ratio of the SUG group was significantly higher than that of PRO and FIB group, the acetic acid/butyric acid ratio of the FAT group was significantly higher compared with the FIB group. In addition, the values of acetic acid/butyric acid ratio in the FAT group in blood sample were significantly decreased compared with those in the PRO group (Fig. 2D). As presented in Fig. 2E, the acetic acid/propionic acid ratio in the FAT group was significantly higher in the fecal samples than the FIB and SUG group, but was lower in the blood samples compared with that of the PRO and SUG group. The ileocecal acetic acid/propionic acid ratio exhibited no differences between the groups.

Amino acids. As presented in Fig. 2F, the total content of amino acids in the feces samples differed among the five groups in the following order: $\mathrm{FIB}<\mathrm{FAT}<\mathrm{CON}<\mathrm{SUG}<\mathrm{PRO}$, and the levels in the FIB group were significantly lower than 
those in the other groups. The PRO group exhibited significantly higher levels than those in the other groups in the feces and ileocecal contents (Fig. 2F and G). The blood samples of the PRO group had significantly lower levels than those of the other groups (Fig. 2H). The total branched-chain amino acid levels, including leucine, isoleucine and valine, in the feces samples of the PRO group were significantly higher than those in CON, FIB and FAT groups (Fig. 2I). Furthermore, there was no significant difference among the five groups in the amino acid levels in the ileocecal content (Fig. 2J). However, the levels of the PRO group were significantly decreased than FIB, CON and FAT group in the blood samples (Fig. 2K).

Leptin and ghrelin production in different tissues. The above results demonstrate the differences in intestinal metabolic products between the various diet groups, but the mechanisms by which the diets affect food control and energy metabolism have remained elusive. Therefore, the expression levels of the appetite control factors leptin and ghrelin were examined in different tissues from the rats. Fig. 3A presents the expression levels of leptin in the adipose, stomach, small intestine, colon and hypothalamus tissues determined using RT-qPCR. The mRNA expression levels of leptin in the adipose tissue were higher than those in the other tissues, and were consistent with the body weight and amount of fat mass in the various groups (FAT $>$ FIB $>$ SUG $>$ CON $>$ PRO). The FAT group had the highest level of leptin than the all other groups). The PRO group had the lowest levels, which differed significantly from those in the FIB and SUG group. In the stomach, small intestine, colon and hypothalamus tissues, the leptin mRNA expression of the FAT group was relatively high, but no significant differences were observed between the groups. Fig. 3B presents the leptin receptor mRNA expression levels in different tissues of the five groups. In the adipose tissue, the expression level was higher than that in the other tissues assessed, and the PRO group had the highest level among the five groups; however, no statistically significant difference was observed between the groups. In the hypothalamus tissue, the PRO group had the lowest level of leptin receptor, and there was a significant difference compared with the all other groups. There were no differences in leptin receptor expression among the groups in the stomach, small intestine and colon tissues.

Fig. 3C and D present the mRNA expression levels of ghrelin and ghrelin receptor in all of the tissues analyzed. The level in the hypothalamus tissue was significantly higher in the PRO group than it was in CON and FAT group; The ghrelin receptor mRNA expression level of the PRO group was higher compared with the CON group. The levels of ghrelin receptor in the other tissues did not differ between the groups.

\section{Discussion}

The present study aimed to investigate the changes in expression levels of metabolite and appetite control factors caused by different diets. The present study reported that a 4 -week unrestricted diet of different compositions affected the obesity-associated metabolic index, indicating that the dietary composition is highly linked to the occurrence of obesity. All of the rats in the present study were fed ad libitum and the actual food intake per rat was not quantified. In order to avoid the influence of diet, the experimental diets were designed based on the percentage of energy of nutrients; for instance, the percentage of energy of the casein content in all diets except for the high-protein diet was $19.2 \%$. The casein content was actually equivalent between all diets. Furthermore, the micronutrient content was almost equivalent between the diets. All diets of the present study had an equivalent energy density of $\sim 3,810 \mathrm{kcal} / \mathrm{kg}$, except for the high-fat diet $(5,179 \mathrm{kcal} / \mathrm{kg})$. This was due to the equal weight of fat having higher calories. Consistent with previous studies, the present study demonstrated that the SD rats fed the high-fat diet successfully developed obesity with disordered glucolipid metabolism. The high-fat diet has been widely used to establish obesity models and has been recognized by domestic and foreign studies (15-17). In a previous study by our group rats on a high-fat diet developed fatty liver after 12 weeks (18). At present, there is limited evidence regarding the pathophysiological effects of a high-protein diet. Numerous studies have proven that a high-protein diet is beneficial for weight loss and improving glucolipid metabolism (19). Wang et al (20) reported that male rats switched to a high-protein diet for 6 weeks after high fat and sugar for 12 weeks had reduced body fat, as well as restored glucose homeostasis and cholecystokinin sensitivity. One study on Mexican adults with metabolic syndrome on hypocaloric diets indicated that a diet with elevated protein ( $1.34 \mathrm{~g} / \mathrm{kg}$ body weight) is associated with significant weight loss compared with a standard protein diet $(0.8 \mathrm{~g} / \mathrm{kg})$, but there were no differences between the groups in terms of glucose, insulin and triglycerides (21). Furthermore, according to certain clinicians, a high-protein diet may overload the body and cause damage to the organs directly involved in protein metabolism and excretion (22). The present study indicated that the SD rats on a high-protein diet exhibited significant weight loss, but also had a markedly disordered glucolipid metabolism. The different effects of a high-protein diet on glucolipid metabolism may depend on the protein type and content in the diet (the percentage of energy derived from protein was $60 \%$ in the present study). The nutritional importance of different types of carbohydrate and their distinct physiological effects on health have remained to be fully elucidated. The role of a high-carbohydrate diet in obesity development has been debated. In the present study, the SD rats on a high-sugar diet or a high-fibre diet exhibited no significant weight increases or loss compared with those in the CON group. The high-sugar and high-fibre diets led to impaired glucose tolerance, while the high-sugar diet also caused impaired fasting glucose compared with the normal control diet. In the present study, certain expected results, including a high-fibre diet reducing weight and improving glucose and lipid metabolism (23-25), were not obtained. When comparing the fibre group with the control group, the high-fibre group exhibited a relatively high dietary fibre content, but the soluble fibre content was not very high, and this groups exhibited a higher glycemic index (GI). Studies conducted with 48,631 European Prospective into Cancer and Nutrition study participants, with a 5.5-year follow-up time, have indicated a positive association between GI and obesity measures (26). A systematic review and meta-analysis of the effects of GI on rodent metabolism demonstrated that a high-GI diet fed to male mice and rats increases body weight, adiposity and fasting 
insulin levels (27). Another study pointed out that consuming a single high-fibre food source was not sufficient to achieve a high-fibre diet in Polish adolescents, but that the consumption of a wide variety of dietary fibre sources, including relatively high-fibre and low-fibre food, may help Polish adolescents achieve a relatively high-fibre diet (28). Overall, the present results suggest that a high-fat diet was positively associated with the occurrence of obesity and that a high-protein diet was associated with a reduced body weight, but also led to glucose and lipid metabolism disorders. In comparison with the control, there was no significant increase in body weight in the high-sugar and high-fibre diet groups after 4 weeks, and the effect of high-sugar and high-fibre diet on glucose and lipid control may depend on the GI and fibre content.

Previous studies have indicated that diet is involved in the pathogenesis of obesity and glucolipid metabolism. Diet is a major factor that modulates the composition and activity of the gut microbiota (10). To date, little is known regarding how diet and intestinal bacteria affect cellular health. SCFAs and amino acids are important metabolites produced by gut microbiota. Recent studies have indicated that excessive SCFAs produced by a particular gut microbiota represent an additional energy source and simultaneously participate in glucose-stimulated insulin secretion from pancreatic $\beta$-cells. These effects are mediated by the interaction of SCFAs with the free fatty acid 2 (FFA2) and FFA3 receptors and the release of peptide hormones, which control appetite (29). SCFAs are thought to inhibit the accumulation of fat in adipose tissue, and therefore, decreased levels may contribute to obesity (30). The present study demonstrated that SD rats on a high-fat diet had lower SCFA levels and that a high-protein diet is associated with higher total SCFA levels in feces. This observation may reflect higher satiety and diet-induced thermogenesis when the dietary protein content is increased. The literature has reported that SCFA butyrate emerged is a significant mediator of health, through its effects on inflammation and satiety (31). Furthermore, the results of the present study confirmed that the SD rats fed the high-fat diet produced less SCFAs, but the percentage of the three SCFAs (acetic acid, propionic acid and butyric acid vs. total SCFAs) was significantly elevated in the feces and blood samples. In addition, the FAT group of the present study had higher acetic acid/propionic acid ratios in their fecal samples. Therefore, it may be inferred that bacteria that produce a higher acetic/propionic ratio may promote the development of obesity. The results of the present study indicate that the high-protein diet resulted in higher amino acid levels in the feces and ileocecal content. The PRO group consumed a much higher proportion of protein than the other groups, and the results indicated that the high protein-based calorie intake was beneficial for reduction of weight gain. Fermentation of dietary proteins may also contribute to SCFA production, but typically provides branched-chain fatty acids, including isobutyrate, 2-methylbutyrate and isovalerate, which originate exclusively from branched-chain amino acids, including valine, isoleucine and leucine. It has been reported that protein- or fat-rich diets supplemented with dietary fibre promote the restoration of beneficial microbes, which may increase SCFA production and inhibit toxin production (32).

The homeostatic control of energy balance is highly regulated by a complex neuronal network where the hypothalamus has a key role in connecting the brainstem and various forebrain regions. There are various peripheral anorexigenic hormones, including leptin, which increase satiety, whereas ghrelin induces hunger. The effect of the microbiota-gut-brain axis is becoming increasingly recognized in psychological and psychiatric disorders (33). In the present study, the levels of the appetite control factors leptin and ghrelin were compared between the groups. In order to avoid the influence of diet itself, the experimental diets were designed based on the percentage of energy of nutrients, for instance, the percentage of energy from protein (casein) was $60 \%$ in the high-protein diet. The percentage of energy from the casein content in all other diets was $19.2 \%$. The micronutrient content was also almost equivalent between diets. Certain studies have pointed out that the energy density also influenced the food intake $(34,35)$. All diets in the present study had an equivalent energy density, except for the high-fat diet. The results of the present study indicated differences in intestinal metabolic products between the different diet groups and differences in leptin and ghrelin expression in different tissues. In addition to serving as an energy source, acetate, propionate and butyrate have been recognized as ligands of the $G$ protein-coupled receptors FFAR2 and FFAR3 (36). Activation of FFAR2 in adipocytes triggers the release of leptin $(37,38)$. Consistent with the literature, the SD rats of the present study fed the high-fat diet had markedly higher leptin levels and it was noted that leptin mRNA was expressed mainly in adipose tissues. Furthermore, leptin levels were positively increased in parallel with the body weight and fat mass size, and in the digestive tract, the leptin mRNA expression of the FAT group was relatively high. It appears that bacteria, which produce higher acetic/propionic and acetic/butyric ratios, may affect the expression of leptin and leptin receptor, which promotes the development of obesity.

Furthermore, the results of the present study indicated that the PRO group had higher ghrelin mRNA expression levels in the hypothalamus tissues compared with that in other diet groups The amino acid composition and digestibility of proteins, which are affected by its source and amount of intake, have a pivotal role in determining the microbiota. Elevated systemic concentrations of certain amino acids, particularly the aromatic and branched-chain amino acids, have been demonstrated to modulate the development of insulin resistance and type 2 diabetes mellitus. Changes in the microbiota may affect the gut barrier and the immune system by regulating gene expression in relevant signaling pathways and regulating the secretion of metabolites (39).

In conclusion, the present study demonstrated that diet has an important role in the development of obesity. The limited sample size of the present study may have been a limitation by hampering the detection of other significant associations, and the effect of metabolites on the regulation of dietary factors, which may be elucidated in cell-based experiments. The lack of other measurements, including the determination of factors from the gut microbiota that induce obesity and metabolic disease, e.g. endotoxin, is another limitation of the present study. Due to time and cost constraints, no further intervention experiments were included. It may be proposed that manipulation of intestinal metabolites by dietary alterations may be a useful strategy to prevent or treat obesity and 
associated complications in the future. It is worth pointing out the physiological diet of rats, as well as their metabolism, are not completely superimposable to those of humans.

\section{Acknowledgements}

Not applicable.

\section{Funding}

This study was supported by the Natural Science Foundation of Zhejiang Province (grant no. LQ15H050002), Projects of Traditional Chinese Medicine in Zhejiang Province (grant no. 2015ZQ004) and Projects of Medical and Health Technology Development Program in Zhejiang Province (grant no. 2016KYA015; 2017KY012; 2018KY266).

\section{Availability of data and materials}

All data generated or analyzed during the present study are included in this published article.

\section{Authors' contributions}

BL and WZh designed the experiments. YL, WZo and WZh performed the experiments and calculations. YL and WZo wrote and edited the manuscript. All authors read and approved the final manuscript.

\section{Ethics approval and consent to participate}

The present study was approved by The Ethics Committee of the Second Xiangya Hospital of Central South University (Changsha, China).

\section{Patient consent for publication}

Not applicable.

\section{Competing interests}

The authors declare that they have no competing interests.

\section{References}

1. Seganfredo FB, Blume CA, Moehlecke M, Giongo A, Casagrande DS, Spolidoro JVN, Padoin AV, Schaan BD and Mottin CC: Weight-loss interventions and gut microbiota changes in overweight and obese patients: A systematic review. Obes Rev 18: 832-851, 2017.

2. Ubeda C, Djukovic A and Isaac S: Roles of the intestinal microbiota in pathogen protection. Clin Transl Immunology 6: e128, 2017.

3. Matsumoto M, Kibe R, Ooga T, Aiba Y, Kurihara S, Sawaki E, Koga Y and Benno Y: Impact of intestinal microbiota on intestinal luminal metabolome. Sci Rep 2: 233, 2012

4. Li Z Quan G, Jiang X, Yang Y, Ding X, Zhang D, Wang X, Hardwidge PR, Ren W and Zhu G: Effects of metabolites derived from gut microbiota and hosts on pathogens. Front Cell Infect Microbiol 8: 314, 2018.

5. Lucas S, Omata Y, Hofmann J, Böttcher M, Iljazovic A, Sarter K, Albrecht O, Schulz O, Krishnacoumar B, Krönke G, et al: Short-chain fatty acids regulate systemic bone mass and protect from pathological bone loss. Nat Commun 9: 55,2018 .
6. Haghikia A, Jörg S, Duscha A, Berg J, Manzel A, Waschbisch A, Hammer A, Lee DH, May C, Wilck N, et al: Dietary fatty acids directly impact central nervous system autoimmunity via the small intestine. Immunity 44: 951-953, 2016.

7. D'Souza WN, Douangpanya J, Mu S, Jaeckel P, Zhang M, Maxwell JR, Rottman JB, Labitzke K, Willee A, Beckmann $\mathrm{H}$, et al: Differing roles for short chain fatty acids and GPR43 agonism in the regulation of intestinal barrier function and immune responses. PLoS One 12: e0180190, 2017.

8. Ojeda P, Bobe A, Dolan K, Leone V and Martinez K: Nutritional modulation of gut microbiota-the impact on metabolic disease pathophysiology. J Nutr Biochem 28: 191-200, 2016.

9. DeGruttola AK, Low D, Mizoguchi A and Mizoguchi E: Current understanding of dysbiosis in disease in human and animal models. Inflamm Bowel Dis 22: 1137-1150, 2016.

10. Kverka $M$ and Tlaskalova-Hogenova $H$ : Intestinal microbiota: Facts and fiction. Dig Dis 35: 139-147, 2017.

11. Birch JM, Ullman K, Struve T, Agger JF, Hammer AS, Leijon $M$ and Jensen HE: Investigation of the viral and bacterial microbiota in intestinal samples from mink (Neovison vison) with pre-weaning diarrhea syndrome using next generation sequencing. PLoS One 13: e0205890, 2018.

12. Ji W, Zhu Y, Kan P, Cai Y, Wang Z, Wu Z and Yang P: Analysis of intestinal microbial communities of cerebral infarction and ischemia patients based on high throughput sequencing technology and glucose and lipid metabolism. Mol Med Rep 16: 5413-5417, 2017.

13. Arocho A, Chen B, Ladanyi M and Pan Q: Validation of the 2-DeltaDeltaCt calculation as an alternate method of data analysis for quantitative PCR of BCR-ABL P210 transcripts. Diagn Mol Pathol 15: 56-61, 2006.

14. Morsczeck C, Korenkov M, Nagelschmidt M, Feher D and Schierholz JM: Total RNA-isolation of abdominal hernia of rats for quantitative real-time reverse transcription (RT) PCR assays. Prep Biochem Biotechnol 38: 87-93, 2008.

15. Alcock J and Lin HC: Fatty acids from diet and microbiota regulate energy metabolism. F1000Res 4: 738, 2015.

16. Candido FG, Valente FX, Grzeskowiak LM, Moreira APB, Rocha DMUP and Alfenas RCG: Impact of dietary fat on gut microbiota and low-grade systemic inflammation: Mechanisms and clinical implications on obesity. Int J Food Sci Nutr 69: 125-143, 2018.

17. de Pinho L, Andrade JM, Paraíso A, Filho AB, Feltenberger JD, Guimarães AL, de Paula AM, Caldeira AP, de Carvalho Botelho AC, Campagnole-Santos MJ and Sousa Santos SH: Diet composition modulates expression of sirtuins and renin-angiotensin system components in adipose tissue. Obesity (Silver Spring) 21: 1830-1835, 2013.

18. Liu JP, Zou WL, Chen SJ, Wei HY, Yin YN, Zou YY and Lu FG: Effects of different diets on intestinal microbiota and nonalcoholic fatty liver disease development. World J Gastroenterol 22: 7353-7364, 2016.

19. Araujo JR, Tomas J, Brenner C and Sansonetti PJ: Impact of high-fat diet on the intestinal microbiota and small intestinal physiology before and after the onset of obesity. Biochimie 141: 97-106, 2017.

20. Wang L, Jacobs JP, Lagishetty V, Yuan PQ, Wu SV, Million M, Reeve JR Jr, Pisegna JR and Taché Y: High-protein diet improves sensitivity to cholecystokinin and shifts the cecal microbiome without altering brain inflammation in diet-induced obesity in rats. Am J Physiol Regul Integr Comp Physiol 313: R473-R486, 2017.

21. Campos-Nonato I, Hernandez L and Barquera S: Effect of a high-protein diet versus standard-protein diet on weight loss and biomarkers of metabolic syndrome: A randomized clinical trial. Obes Facts 10: 238-251, 2017.

22. da Rosa Lima T, Ávila ETP, Fraga GA, de Souza Sena M, de Souza Dias AB, de Almeida PC, Dos Santos Trombeta JC, Junior RCV, Damazo AS, Navalta JW, et al: Effect of administration of high-protein diet in rats submitted to resistance training. Eur J Nutr 57: 1083-1096, 2018.

23. Choi Y, Giovannucci E and Lee JE: Glycaemic index and glycaemic load in relation to risk of diabetes-related cancers: A meta-analysis. Br J Nutr 108: 1934-1947, 2012.

24. Huang J, Fang YJ, Xu M, Luo H, Zhang NQ, Huang WQ, Pan ZZ, Chen YM and Zhang CX: Carbohydrate, dietary glycaemic index and glycaemic load, and colorectal cancer risk: A case-control study in China. Br J Nutr 119: 937-948, 2018.

25. Dong JY, Zhang L, Zhang YH and Qin LQ: Dietary glycaemic index and glycaemic load in relation to the risk of type 2 diabetes: A meta-analysis of prospective cohort studies. Br J Nutr 106: 1649-1654, 2011. 
26. Romaguera D, Angquist L, Du H, Jakobsen MU, Forouhi NG, Halkjaer J, Feskens EJ, van der A DL, Masala G, Steffen A, et al: Dietary determinants of changes in waist circumference adjusted for body mass index-a proxy measure of visceral adiposity. PLoS One 5: e11588, 2010.

27. Campbell GJ, Senior AM and Bell-Anderson KS: Metabolic effects of high glycaemic index diets: A systematic review and meta-analysis of feeding studies in mice and rats. Nutrients 9: E646, 2017.

28. Krusinska B, Kowalkowska J, Wadolowska L, Wuenstel JW, Slowinska MA and Niedzwiedzka E: Fibre-related dietary patterns: Socioeconomic barriers to adequate fibre intake in polish adolescents. A short report. Nutrients 9: E590, 2017.

29. Murugesan S, Nirmalkar K, Hoyo-Vadillo C, Garcia-Espitia M, Ramirez-Sanchez D and Garcia-Mena J: Gut microbiome production of short-chain fatty acids and obesity in children. Eur J Clin Microbiol Infect Dis 37: 621-625, 2018.

30. Tilg H and Moschen AR: Microbiota and diabetes: An evolving relationship. Gut 63: 1513-1521, 2014.

31. Lu Y, Fan C, Li P, Lu Y, Chang X and Qi K: Short chain fatty acids prevent high-fat-diet-induced obesity in mice by regulating $\mathrm{G}$ protein-coupled receptors and gut microbiota. Sci Rep 6 : 37589, 2016.

32. Sanchez JI, Marzorati M, Grootaert C, Baran M, Van Craeyveld V, Courtin CM, Broekaert WF, Delcour JA, Verstraete W and Van de Wiele T: Arabinoxylan-oligosaccharides (AXOS) affect the protein/carbohydrate fermentation balance and microbial population dynamics of the simulator of human intestinal microbial ecosystem. Microb Biotechnol 2: 101-113, 2009.

33. van de Wouw M, Schellekens H, Dinan TG and Cryan JF: Microbiota-gut-brain axis: Modulator of host metabolism and appetite. J Nutr 147: 727-745, 2017.
34. Murakami K, Livingstone MB, Okubo H and Sasaki S: Energy density of the diets of Japanese adults in relation to food and nutrient intake and general and abdominal obesity: A cross-sectional analysis from the 2012 national health and nutrition survey, Japan. Br J Nutr 117: 161-169, 2017.

35. Raynor HA and Vadiveloo M: Understanding the relationship between food variety, food intake, and energy balance. Curr Obes Rep 7: 68-75, 2018.

36. Brown AJ, Goldsworthy SM, Barnes AA, Eilert MM, Tcheang L, Daniels D, Muir AI, Wigglesworth MJ, Kinghorn I, Fraser NJ, et al: The Orphan G protein-coupled receptors GPR41 and GPR43 are activated by propionate and other short chain carboxylic acids. J Biol Chem 278: 11312-11319, 2003

37. Blaut M: Gut microbiota and energy balance: Role in obesity. Proc Nutr Soc 74: 227-234, 2015.

38. Xiong Y, Miyamoto N, Shibata K, Valasek MA, Motoike T, Kedzierski RM and Yanagisawa M: Short-chain fatty acids stimulate leptin production in adipocytes through the $G$ protein-coupled receptor GPR41. Proc Natl Acad Sci USA 101: 1045-1050, 2004.

39. Ma N, Tian Y, Wu Y and Ma X: Contributions of the interaction between dietary protein and gut microbiota to intestinal health. Curr Protein Pept Sci 18: 795-808, 2017.

(i) $\ominus$ This work is licensed under a Creative Commons Attribution-NonCommercial-NoDerivatives 4.0 International (CC BY-NC-ND 4.0) License. 